\title{
Early Stages of Ultrafast Spin Dynamics in a $3 d$ Ferromagnet
}

\author{
R. Gort, ${ }^{1 *}$ K. Bühlmann, ${ }^{1}$ S. Däster, ${ }^{1}$ G. Salvatella, ${ }^{1}$ N. Hartmann, ${ }^{2}$ Y. Zemp, ${ }^{1}$ S. Holenstein, ${ }^{3,4}$ \\ C. Stieger, ${ }^{5}$ A. Fognini, ${ }^{6}$ T. U. Michlmayr, ${ }^{1}$ T. Bähler, ${ }^{1}$ A. Vaterlaus, ${ }^{1}$ and Y. Acremann ${ }^{1}$ \\ ${ }^{1}$ Laboratory for Solid State Physics, ETH Zurich, 8093 Zurich, Switzerland \\ ${ }^{2}$ Institute for Quantum Electronics, ETH Zurich, 8093 Zurich, Switzerland \\ ${ }^{3}$ Laboratory for Muon Spin Spectroscopy, Paul Scherrer Institut, 5232 Villigen PSI, Switzerland \\ ${ }^{4}$ Department of Physics, University of Zurich, Winterthurerstrasse 190, CH-8057 Zurich, Switzerland \\ ${ }^{5}$ Department of Information Technology and Electrical Engineering, ETH Zurich, 8092 Zurich, Switzerland \\ ${ }^{6}$ Department of Quantum Nanoscience, TU Delft, 2628 CD Delft, Netherlands
}

(Received 22 February 2018; revised manuscript received 24 July 2018; published 24 August 2018)

\begin{abstract}
Prior to the development of pulsed lasers, one assigned a single local temperature to the lattice, the electron gas, and the spins. With the availability of ultrafast laser sources, one can now drive the temperature of these reservoirs out of equilibrium. Thus, the solid shows new internal degrees of freedom characterized by individual temperatures of the electron gas $T_{e}$, the lattice $T_{l}$ and the spins $T_{s}$. We demonstrate an analogous behavior in the spin polarization of a ferromagnet in an ultrafast demagnetization experiment: At the Fermi energy, the polarization is reduced faster than at deeper in the valence band. Therefore, on the femtosecond time scale, the magnetization as a macroscopic quantity does not provide the full picture of the spin dynamics: The spin polarization separates into different parts similar to how the single temperature paradigm changed with the development of ultrafast lasers.
\end{abstract}

DOI: 10.1103/PhysRevLett.121.087206

The phenomenon of ultrafast demagnetization has been intensively discussed since its discovery in 1996 [1]. In the early years, most of the experiments were conducted by optical means, which led to some controversy regarding the applicability of such methods in the ultrafast regime [2-4]. It could be shown that the magneto-optical methods, indeed, reliably detect the magnetization on the time scale of relevance [5-8]. Magneto-optical methods have been extended to the vacuum ultraviolet range using $M$-edge spectroscopy with high harmonic sources [9-11] and x-ray magnetic circular dichroism studies [12-14]. These methods offer elemental selectivity and the possibility of detecting the spin and orbital moments separately by sum rule analysis [15]. The recent development of timeresolved magnetic ellipsometry also allows for the separation of spin dynamics within different magnetic layers $[16,17]$. To compare the results from different experimental techniques, we typically treat the measured spin polarization as the magnetization. However, this energy integrated electron spin polarization does not necessarily reflect the details of the spin dynamics at different binding energies.

Here, we investigate the dynamics of spin polarization through an ultrafast demagnetization experiment using timeand energy-resolved photoelectron spectroscopy. We observe that the spin polarization near the Fermi energy is reduced within $\approx 60 \mathrm{fs}$, whereas the polarization $2 \mathrm{eV}$ below the Fermi energy decreases within $\approx 450$ fs. This result requires us to consider the energy-integrated spin polarization from a different perspective. We can still define a magnetization as the average magnetic moment of a unit of volume. However, we cannot treat the spin polarization in the conduction band as a uniformly varying, rigid system.

To probe the spin dynamics with femtosecond time resolution, we perform a laser-pump-ultraviolet-probe experiment on a ferromagnetic thin iron film. A pump pulse with a wavelength of $800 \mathrm{~nm}$ (corresponding to a photon energy of $1.5 \mathrm{eV}$ ) excites the sample. Following a variable time delay $t$, the sample is exposed to the probe beam, which emits electrons into vacuum. These electrons are sent through a hemispherical energy analyzer followed by a spin analyzer. The setup consists of an amplified tabletop Ti: Sapphire laser system (Coherent Inc. Legend Elite), delivering pulses of $20 \mathrm{fs} F W H M$ duration at a $10 \mathrm{kHz}$ repetition rate and $800 \mathrm{~nm}$ wavelength. The pulses are split into the pump and probe beams with separate pulse compressor stages. The pump fluence on the sample was set to $1.5 \mathrm{~mJ} / \mathrm{cm}^{2}$. The $21 \mathrm{eV}$ probe beam is generated by a high harmonic source pumped at $400 \mathrm{~nm}$ [18-20]. The $400 \mathrm{~nm}$ beam has a pulse energy of $0.2 \mathrm{~mJ}$ and is focused into an $\mathrm{Ar}$ jet. After a differential pumping stage, the beam hits a $\mathrm{Si}$ wafer at the Brewster angle for $400 \mathrm{~nm}$, significantly reducing the intensity of the copropagating fundamental beam, before being reflected on two curved Bragg mirrors that focus the remaining beam onto the sample and selectively reflect at $21 \mathrm{eV}$. An aluminum foil, acting as a vacuum barrier between the mirror chamber and the ultrahighvacuum measurement chamber, cuts off the low harmonic components. The resulting probe beam has a pulse duration 
that allows for measuring events on a sub $30 \mathrm{fs}$ timescale, as experimentally determined. The bandwidth of the high harmonic pulses gives rise to a reduced energy resolution in the following photoemission process. The sample under investigation is a $20 \mathrm{ML}$ single crystalline iron film that was placed on a W(110) substrate by electron beam evaporation with a rate of $0.01 \mathrm{ML} / \mathrm{s}$ [21]. The substrate was annealed in an oxygen atmosphere of $4.5 \times 10^{-8} \mathrm{mbar}$ for $5 \mathrm{~min}$ and subsequently flashed three times before $\mathrm{Fe}$ deposition. A hemispherical energy analyzer disperses the photoemitted electrons. After the output of the energy analyzer, we use spin-polarized low-energy electron diffraction spin filtering on an $\operatorname{Ir}(001)$ crystal capped with one monolayer of gold to obtain spin information $[22,23]$. After reflection on the spin filter crystal, the electron distribution is amplified by a microchannel plate and detected on a phosphor screen followed by a CCD camera. The image on the detector encodes the kinetic energy on the $x$ axis and the emission angle on the $y$ axis. Spin information is contained in the reflectivity of the crystal. The images are digitally corrected for distortion caused by imperfections of the electron optics. The magnetization of the sample $M_{\uparrow, \downarrow}$ is reversed by the stray field of a pulsed coil [24]. To determine the spin polarization, the asymmetry between a pair of images for $M_{\uparrow, \downarrow}$ is calculated and normalized by a reference image containing the Sherman factor for each pixel. This "Sherman image" is determined from cascade electrons of iron with a kinetic energy of $1 \mathrm{eV}$. In this case, the sample is exposed to the beam of an electron gun with a primary electron energy of $1.5 \mathrm{keV}$ [25]. To obtain spin-resolved spectra, we integrate over the $y$ axis of the images and subtract an energy-independent background, obtained at energies above the Fermi level in an unpumped state, according to [19].

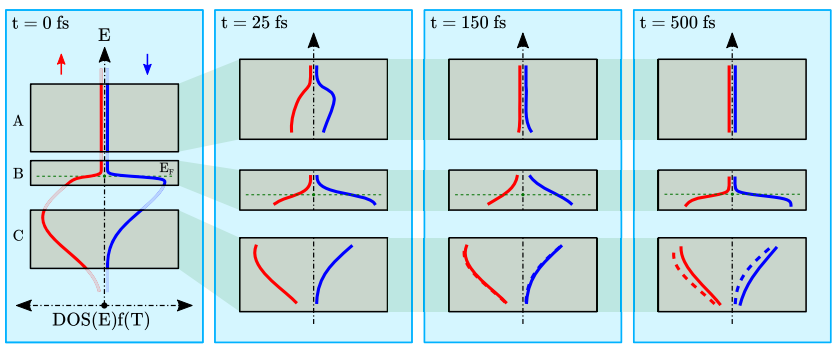

FIG. 1. Schematic representation of our findings. Initially, the electron gas is in thermal equilibrium. For both spin directions, all states below $E_{F}$ are filled (shown in row C). The Fermi-Dirac distribution cuts off the filling of states shown in row B (the green dashed line represents $E_{f}$ ). Row A shows the initially unoccupied states above $E_{F}$. Note that 25 fs after the pump pulse, we observe nonthermal electrons above $E_{F}$, which represent the spin polarization from where they originate. These electrons relax into a hot Fermi-Dirac distribution. However, only $\approx 500$ fs later, we also observe a loss of spin polarization within the filled states in the conduction band, which is caused by a modification of the spinsplit DOS. The dashed lines represent the ground state DOS.
The schematic representation in Fig. 1 provides an overview of our findings. The top row shows the electron density of the originally empty states as a function of time. The middle row presents the situation at $E_{F}$, and the bottom row shows the valence band carrier density. With our experiment, we are able to experimentally observe these regions separately. The initial state consists of the spin-split density of states (DOS) filled with electrons to the Fermi energy $E_{F}$. The electron gas is excited by the pump pulse as the electric field of the laser couples to the charge of the electrons. The pump laser pulse causes transitions from the filled states below $E_{F}$ to the empty states above $E_{F}$. These transitions are elastic, vertical, and expected to conserve the spin. The electron distribution during the pump pulse is nonthermal: The occupation of states cannot be described by a Fermi-Dirac function. These nonthermal electrons thermalize to a Fermi-Dirac distribution of elevated temperature [26]. The details of the relaxation process of nonthermal electrons have been investigated using spin resolved two photon photoemission by Cinchetti et al. [27]. On the same time scale, we observe a reduction in the spin polarization close to the Fermi energy. The origin of this depolarization is still under debate because two contributions are likely relevant: Spins can flip locally and cause demagnetization within the ferromagnet [6]. Additionally, transport effects are also relevant: The high electron gas temperature at the surface of the sample can lead to the generation of a spin current that transports angular momentum from the surface to the bulk or even into the substrate, where spin flips can occur outside the view of the observer [28]. This aspect may become increasingly important for generating strong and short spin current pulses.

Experimentally, we can only probe a fraction of the Brillouin zone (BZ) due to the limited angular acceptance. The [110] direction of the crystal is oriented towards the energy analyzer. The magnetization direction of our samples points along the [110] direction [21], which is perpendicular to the angular sensitive axis of our detector, that lies in the mirror plane of the incoming laser beams. We integrate over an angular range of $\pm 4^{\circ}$, which corresponds to $k_{\|}= \pm 0.15 \AA^{-1}$. The fixed photon energy of $21 \mathrm{eV}$ probe states close to the $\Gamma$ point within the neighboring $\mathrm{BZ}$ at $k_{\perp}=0.16 \AA^{-1}$ in direction $\Gamma N$. Both pump and probe beam are $p$ polarized. Therefore, the symmetry properties of matrix elements with respect to our experimental geometry allow for detection of the $d_{x y}$ minority states and the $d_{z^{2}}$ majority states $[29,30]$.

In Fig. 2, we show the measured electron spectra for the majority and minority electrons for different pump-probe delays. The energy axis has been calibrated such that the Fermi energy $E_{F}$ corresponds to $0 \mathrm{eV}$. For a negative pumpprobe delay $t$, we see the unperturbed electron spectra $I_{\uparrow, \downarrow}$ of the form 


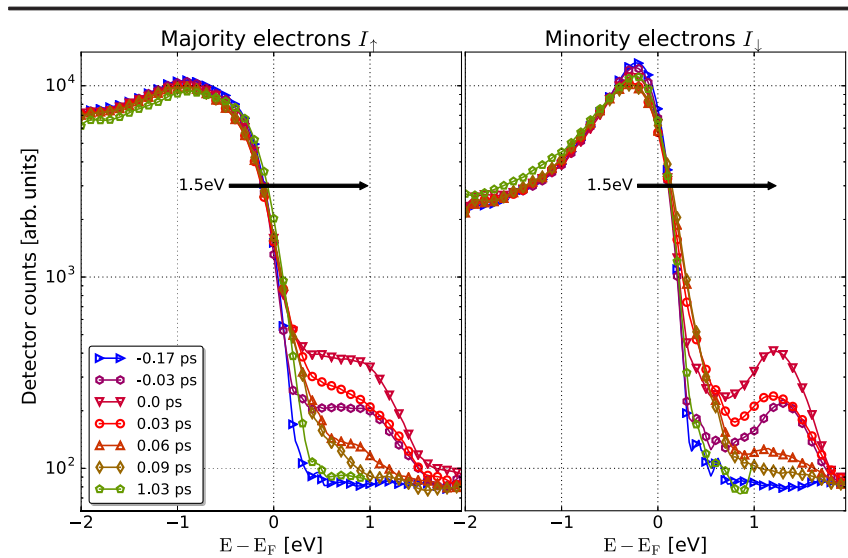

FIG. 2. Number of detected minority and majority electrons $I_{\uparrow, \downarrow}(E, t)$. Note that the raw detector signals have been corrected by the Sherman function of the spin polarimeter. The nonthermal electrons are caused by elastic, vertical transitions from the valence band to the empty states above $E_{F}$. These excitations decay into a Fermi-Dirac distribution of elevated temperature. For better visibility, a Savitzky-Golay filter has been applied to the data.

$$
I_{\uparrow, \downarrow} \propto\left[D_{\uparrow, \downarrow}(E) f\left(E, \mu_{\uparrow, \downarrow}, T\right)\right] * R(E),
$$

with the joint density of states (JDOS) $D_{\uparrow, \downarrow}$ and the FermiDirac function $f$, which depends on the spin-dependent chemical potentials $\mu_{\uparrow, \downarrow}(T)$ and the temperature $T . R(E)$ represents the point-spread function of the instrument. Note that, in equilibrium, $\mu_{\uparrow}=\mu_{\downarrow} \approx E_{F}$. Matrix elements are taken into account implicitly by the selection of allowed transitions for $D$.

For the minority electrons, there is a peak near $E_{F}$ in the JDOS. The drop-off at $E_{F}$ is caused by the Fermi-Dirac function. The JDOS for the majority electrons drops before $E_{F}$. This leads to an inversion of the spin polarization at $E_{F}$, as shown in Fig. 3. Deeper within the valence band, the polarization shows majority character, whereas the polarization at $E_{F}$ shows minority character.

As the electron gas is excited, we observe the early steps in ultrafast demagnetization. The pump laser pulse (photon energy: $\hbar \omega_{p}=1.5 \mathrm{eV}$ ) directly excites the states of the energy interval $\left[E_{F}-\hbar \omega_{p}, E_{F}\right]$ to the empty states above $E_{F}$ by vertical, elastic transitions. The shape of the JDOS below $E_{F}$ is, therefore, imprinted onto the spectrum above $E_{F}$. Within $100 \mathrm{fs}$, these nonthermal electrons decay into a Fermi-Dirac distribution of elevated temperature.

In Fig. 3(a), we show the polarization $P(E, t)$ as a function of the electron energy $E$ and time $t$. The nonthermal electrons show the polarization of their origin. At this time, the polarization deeper below $E_{F}$ has not yet been altered by the pump pulse. Figure 3(c) shows the change of the polarization for $0.6 \mathrm{eV}$ above $E_{F}$. The sign of $P(t, E=$ $0.6 \mathrm{eV}$ ) reverses at $t \approx 50 \mathrm{fs}$. This reversal is caused by the broadening of the Fermi edge, providing electrons of minority character at $E=0.6 \mathrm{eV}$, as well as the relaxation
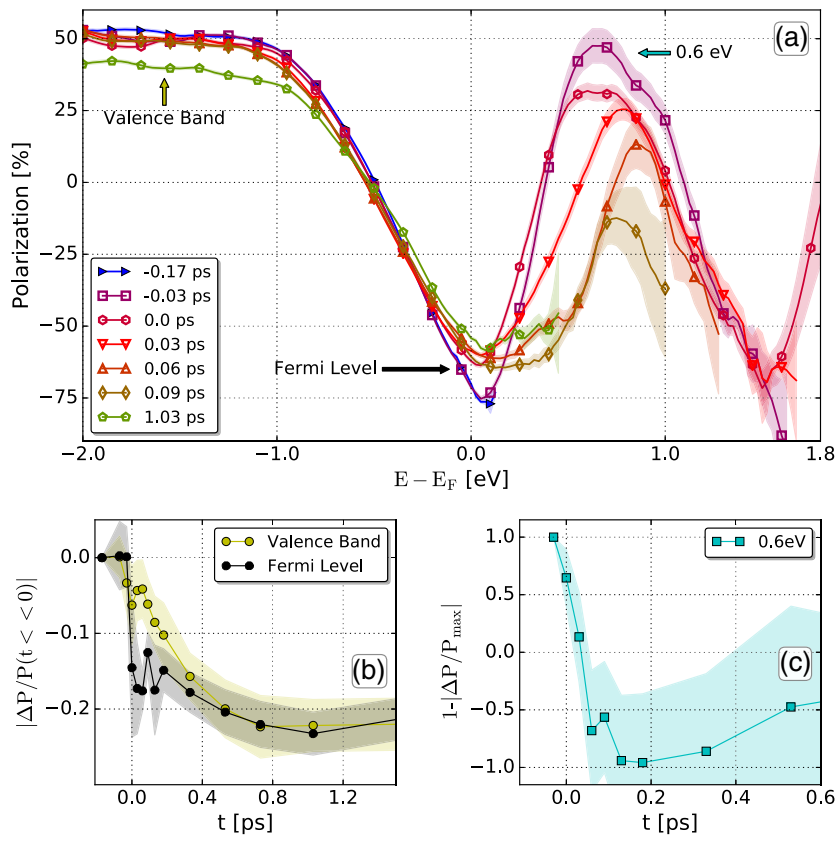

FIG. 3. (a) Energy-dependent polarization $P(E, t)$ for the entire measured energy range. The polarization at the Fermi energy is reversed compared to the valence band. The pump pulse excites electrons from the energy interval $\left[E_{F}-1.5 \mathrm{eV}, E_{F}\right]$ to the interval $\left[E_{F}, E_{F}+1.5 \mathrm{eV}\right]$. These electrons maintain their initial polarization. The data have been filtered by a Savitzky-Golay filter. We plot points where the signal to noise ratio for the filtered data is $>2$. For a set of selected time delays, we also show a region of error. (b) The polarization at $E_{F}$ decreases within $\approx 60 \mathrm{fs}$. In contrast, the polarization of the valence electrons decays significantly slower. (c) At $0.6 \mathrm{eV}$ above the Fermi energy, one can observe a reversal of the polarization. Initially, the nonthermal electrons have majority character as they originate from below the Fermi energy. As the carriers relax and the Fermi edge broadens, the polarization is dominated by the minority carriers at $E_{F}$.

of the nonthermal electrons. The relaxation of nonthermal electrons has been investigated previously by Cinchetti et al. [27] using spin resolved two photon photoemission. They observe more complex refilling dynamics involving spin flips from minority to majority states. Here, we cannot observe these transitions, as the thermal broadening of the Fermi edge masks such effects.

In Fig. 3(b), we plot the time evolution of the normalized polarization change $|\Delta P(t) / P(t \ll 0)|$ at $2.0 \mathrm{eV}$ below $E_{F}$ (yellow) compared to the polarization at $E_{F}$ (black). At $E_{F}$, a standard fit routine reveals a demagnetization time of $60 \mathrm{fs}$. In contrast, at $E_{F}-2.0 \mathrm{eV}$, the same analysis leads to a significantly longer demagnetization time of $450 \mathrm{fs}$. Therefore, the valence band polarization cannot be treated as a rigid, homogeneous system: The spin dynamics depends on the binding energy of the electrons. The depolarization is faster, where the fundamental processes for demagnetization occur. Both spin flips and spin currents are present close to the Fermi energy or above: These processes require empty final states. Only later does the 
spin polarization of the remainder of the valence band follow the depolarization observed at $E_{F}$.

A reduction in the polarization significantly below the Fermi edge can only be achieved if the DOS changes. All states are filled for $E \ll E_{F}$. There are two mechanisms discussed in the literature. There can be a reduction in the exchange splitting acting as a driving force for the demagnetization [31]. Another possibility is to assume spatial inhomogeneities of the magnetization direction caused by the emission of spin waves. Because our photoemission experiment does not provide any spatial resolution, the resulting spin-split DOS would represent a mixing of the DOS for the majority with the minority electrons. This so-called band structure mirroring has been observed previously [32].

From the measured data, we determine the JDOS $D_{\uparrow, \downarrow}(t)$ : We divided the measured intensities by a convolution of the resolution and the Fermi distribution. The input parameters $T(t), \mu_{\uparrow, \downarrow}(t)$ and $\Delta E$ have been determined in a self-consistent way using the same data. $D_{\uparrow, \downarrow}$ is depicted in Fig. 4(a). If the band structure mirroring is correct, then the spin-split JDOS is determined by the JDOS before the pump laser pulse

$$
D_{\uparrow, \downarrow}(t, E)=[1-\alpha(t)] D_{\uparrow, \downarrow}^{0}+\alpha(t) D_{\downarrow, \uparrow}^{0} .
$$

Here, $D^{0}$ denotes the unperturbed JDOS, and $\alpha(t)$ is a dimensionless parameter describing the mixing between majority and minority states

$$
\alpha(t)=\frac{1}{2} \frac{\Delta P(t)}{P(t \ll 0)} .
$$

In Fig. 4(b), we show the JDOS before $t_{0}$ reconstructed from the time-dependent measurements by inverting the band structure mirroring effect; see Eq. (2). The result is consistent with the findings of S. Eich et al. [32]. The observed changes of the band structure in the valence band

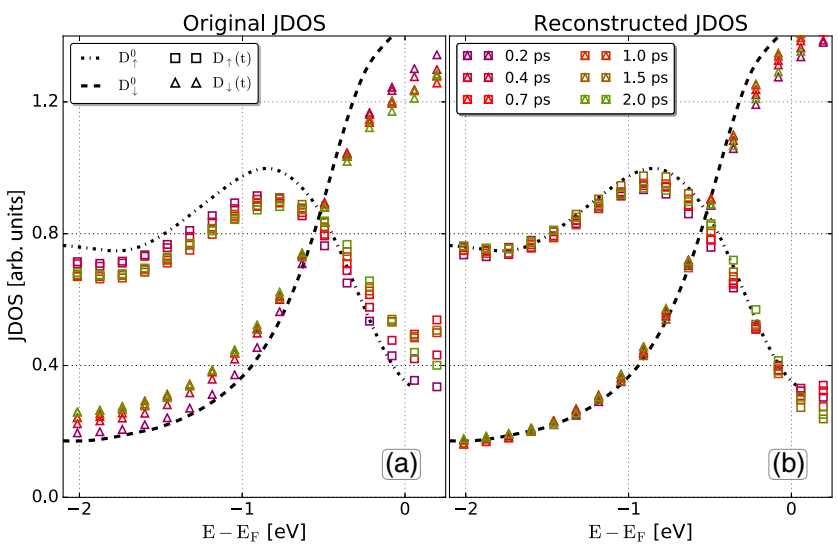

FIG. 4. (a) The density of states $D_{\uparrow, \downarrow}$ as a function of the electron energy and the pump-probe delay. (b) The reconstruction of the unperturbed density of states by assuming the band structure mirroring effect [32]. can be explained by spatial inhomogeneities of the magnetization. Such inhomogeneities can be caused by spin waves. Averaging the different regions by the nonspatially resolved experiment directly leads to the observed mixing between the majority and minority JDOS.

This band structure mirroring effect indicates that delocalized excitations are relevant for the depolarization within the valence band. Such excitations can be caused by any thermal fluctuations induced by the pump laser pulse and are not necessarily tied to the nonthermal spin population above $E_{f}$. This has been demonstrated by [13,33]: A ferromagnet can be demagnetized on the femtosecond time scale by the heat transport through a nonmagnetic metal film alone. This transport can be purely thermal [33]. Therefore, the spin polarization within the nonthermal electron distribution shortly after the laser pulse is not required to communicate with the rest of the spin system in order to cause demagnetization in the valence band.

S. Eich et al. [32] identify the spin transport as the driving force for the generation of spin waves and, therefore, the band structure mirroring effect. The details of the matching between the Fermi surface of the substrate and the majority and minority Fermi surfaces of the ferromagnet determine the spin transport properties at the interface. This could explain the difference between the dynamics in $\mathrm{Co}$ on $\mathrm{Cu}$ and $\mathrm{Fe}$ on $\mathrm{W}$.

In conclusion, using time- and spin-resolved photoemission, we observe the demagnetization of a thin Fe film. We show that the spin polarization is not uniformly affected by the pump laser beam. Initially, the electrons are directly excited by the pump laser by elastic, vertical transitions. These electrons still possess their original spin polarization. Upon thermalization, we observe that electrons close to the Fermi energy lose their spin polarization within $\approx 60 \mathrm{fs}$, whereas those deeper in the valence band require an order of magnitude more time for depolarization.

The magnetization is still defined at any time of the experiment: It is the average magnetic moment $M=\mu_{B} g<$ $J>_{V}$ for a unit volume $V$. However, the spin polarization cannot be viewed as a rigid structure: It separates into parts that possess their own dynamics. It is conceivable that the transient state of spin imbalance could be utilized for novel approaches for ultrafast and efficient magnetization manipulation.

In our experiment, we could only probe a small part of the whole Brillouin zone. With the development of novel time of flight electron spectrometers in combination with free electron laser sources, it will be possible to map the entire band structure and study the spin dynamics of the complete BZ. The power of such instruments has been shown using synchrotron radiation by Kutnyakhov and co-workers [34] and, likely, will allow for time resolved band structure tomography at free electron laser sources.

This work was supported by the Swiss National Science Foundation (Grant No. 200021 and 156614) and ETH 
Zurich. We thank Professor Gerd Schönhense (University of Mainz, Germany) for fruitful discussions and expertise on the subject of SPLEED spin detectors.

*rgort@phys.ethz.ch

[1] E. Beaurepaire, J.-C. Merle, A. Daunois, and J.-Y. Bigot, Phys. Rev. Lett. 76, 4250 (1996).

[2] B. Koopmans, M. van Kampen, J. T. Kohlhepp, and W. J. M. de Jonge, Phys. Rev. Lett. 85, 844 (2000).

[3] P. M. Oppeneer and A. Liebsch, J. Phys. Condens. Matter 16, 5519 (2004).

[4] K. Carva, M. Battiato, and P. M. Oppeneer, Nat. Phys. 7, 665 (2011).

[5] G. P. Zhang, W. Hubner, G. Lefkidis, Y. Bai, and T. F. George, Nat. Phys. 5, 499 (2009).

[6] B. Koopmans, G. Malinowski, F. Dalla Longa, D. Steiauf, M. Fahnle, T. Roth, M. Cinchetti, and M. Aeschlimann, Nat. Mater. 9, 259 (2010).

[7] A. Weber, F. Pressacco, S. Günther, E. Mancini, P. M. Oppeneer, and C. H. Back, Phys. Rev. B 84, 132412 (2011).

[8] C. La-O-Vorakiat, E. Turgut, C. A. Teale, H. C. Kapteyn, M. M. Murnane, S. Mathias, M. Aeschlimann, C. M. Schneider, J. M. Shaw, H. T. Nembach, and T. J. Silva, Phys. Rev. X 2, 011005 (2012).

[9] C. La-O-Vorakiat, M. Siemens, M. M. Murnane, H. C. Kapteyn, S. Mathias, M. Aeschlimann, P. Grychtol, R. Adam, C. M. Schneider, J. M. Shaw, H. Nembach, and T. J. Silva, Phys. Rev. Lett. 103, 257402 (2009).

[10] S. Mathias, C. La-o-vorakiat, P. Grychtol, P. Granitzka, E. Turgut, J. M. Shaw, R. Adam, H. T. Nembach, M. E. Siemens, S. Eich, C. M. Schneider, T. J. Silva, M. Aeschlimann, M. M. Murnane, and H. C. Kapteyn, Proc. Natl. Acad. Sci. U.S.A. 109, 4792 (2012).

[11] E. Turgut, C. La-o-vorakiat, J. M. Shaw, P. Grychtol, H. T. Nembach, D. Rudolf, R. Adam, M. Aeschlimann, C. M. Schneider, T. J. Silva, M. M. Murnane, H. C. Kapteyn, and S. Mathias, Phys. Rev. Lett. 110, 197201 (2013).

[12] C. Boeglin, E. Beaurepaire, V. Halte, V. Lopez-Flores, C. Stamm, N. Pontius, H. A. Durr, and J.-Y. Bigot, Nature (London) 465, 458 (2010).

[13] A. Eschenlohr, M. Battiato, P. Maldonado, N. Pontius, T. Kachel, K. Holldack, R. Mitzner, A. Föhlisch, P. M. Oppeneer, and C. Stamm, Nat. Mater. 12, 332 (2013).

[14] I. Radu, K. Vahaplar, C. Stamm, T. Kachel, N. Pontius, H. A. Dürr, T. A. Ostler, J. Barker, R. F. L. Evans, R. W. Chantrell, A. Tsukamoto, A. Itoh, A. Kirilyuk, T. Rasing, and A. V. Kimel, Nature (London) 472, 205 (2011).

[15] C. Stamm, T. Kachel, N. Pontius, R. Mitzner, T. Quast, K. Holldack, S. Khan, C. Lupulescu, E. F. Aziz, M. Wietstruk, H. A. Dürr, and W. Eberhardt, Nat. Mater. 6, 740 (2007).
[16] J. Hamrle, J. Ferré, M. Nývlt, and S. Višňovský, Phys. Rev. B 66, 224423 (2002).

[17] A. J. Schellekens, N. de Vries, J. Lucassen, and B. Koopmans, Phys. Rev. B 90, 104429 (2014).

[18] S. Eich, A. Stange, A. Carr, J. Urbancic, T. Popmintchev, M. Wiesenmayer, K. Jansen, A. Ruffing, S. Jakobs, T. Rohwer, S. Hellmann, C. Chen, P. Matyba, L. Kipp, K. Rossnagel, M. Bauer, M. Murnane, H. Kapteyn, S. Mathias, and M. Aeschlimann, J. Electron Spectrosc. Relat. Phenom. 195, 231 (2014).

[19] M. Plötzing, R. Adam, C. Weier, L. Plucinski, S. Eich, S. Emmerich, M. Rollinger, M. Aeschlimann, S. Mathias, and C. M. Schneider, Rev. Sci. Instrum. 87, 043903 (2016).

[20] G. Rohde, A. Hendel, A. Stange, K. Hanff, L.-P. Oloff, L. X. Yang, K. Rossnagel, and M. Bauer, Rev. Sci. Instrum. 87, 103102 (2016).

[21] S. Miesch, A. Fognini, Y. Acremann, A. Vaterlaus, and T. U. Michlmayr, J. Appl. Phys. 109, 013905 (2011).

[22] M. Kolbe, P. Lushchyk, B. Petereit, H. J. Elmers, G. Schönhense, A. Oelsner, C. Tusche, and J. Kirschner, Phys. Rev. Lett. 107, 207601 (2011).

[23] C. Tusche, A. Krasyuk, and J. Kirschner, Ultramicroscopy 159, 520 (2015).

[24] A. Fognini, T. U. Michlmayr, T. U. Bähler, C. Wetli, A. Vaterlaus, and Y. Acremann, Rev. Sci. Instrum. 83, 063906 (2012).

[25] S. F. Mao, X. Sun, X. W. Fang, B. Da, and Z. J. Ding, Surf. Interface Anal. 44, 703 (2012).

[26] H.-S. Rhie, H. A. Dürr, and W. Eberhardt, Phys. Rev. Lett. 90, 247201 (2003).

[27] M. Cinchetti, M. Sánchez Albaneda, D. Hoffmann, T. Roth, J.-P. Wüstenberg, M. Krauß, O. Andreyev, H. C. Schneider, M. Bauer, and M. Aeschlimann, Phys. Rev. Lett. 97, 177201 (2006).

[28] M. Battiato, K. Carva, and P. M. Oppeneer, Phys. Rev. Lett. 105, 027203 (2010).

[29] J. Schäfer, M. Hoinkis, E. Rotenberg, P. Blaha, and R. Claessen, Phys. Rev. B 72, 155115 (2005).

[30] J. Callaway and C. S. Wang, Phys. Rev. B 16, 2095 (1977).

[31] B. Y. Mueller, T. Roth, M. Cinchetti, M. Aeschlimann, and B. Rethfeld, New J. Phys. 13, 123010 (2011).

[32] S. Eich, M. Plötzing, M. Rollinger, S. Emmerich, R. Adam, C. Chen, H. C. Kapteyn, M. M. Murnane, L. Plucinski, D. Steil, B. Stadtmüller, M. Cinchetti, M. Aeschlimann, C. M. Schneider, and S. Mathias, Sci. Adv. 3, e1602094 (2017).

[33] G. Salvatella, R. Gort, K. Bühlmann, S. Däster, A. Vaterlaus, and Y. Acremann, Struct. Dyn. 3, 055101 (2016).

[34] D. Kutnyakhov, S. Chernov, K. Medjanik, R. Wallauer, C. Tusche, M. Ellguth, S. A. Nepijko, M. Krivenkov, J. Braun, S. Borek, J. Minár, H. Ebert, H. J. Elmers, and G. Schönhense, Sci. Rep. 6, 29394 (2016). 